\title{
Effectiveness of Interventions to Increase Referral of Clients Exhibiting TB Symptoms by Pharmacies and Corner Stores in Santo Domingo, Dominican Republic
}

\author{
Ellen M.H. Mitchell ${ }^{*}, 1,2$, Eddy Pérez-Then ${ }^{3}$, Ivonne Orejel-Juarez ${ }^{4}$, Jeannette Baez ${ }^{3}$, \\ Franz Gonzáles ${ }^{1}$, Ana Lucia Morrobel ${ }^{5}$, Belkys Marcelino ${ }^{6}$ and Netty Kamp ${ }^{1}$
}

\author{
${ }^{I}$ KNCV Tuberculosis Foundation, The Hague, The Netherlands \\ Health \& Development, The Netherlands \\ ${ }^{3}$ Centro Nacional de Investigaciones en Salud Materna Infantil (CENISMI) \\ ${ }^{4}$ National Tuberculosis Program Mexico, Mexico \\ ${ }^{5}$ Dirección Municipal de Área IV de Salud del Distrito Nacional \\ ${ }^{6}$ Dominican Republic National TB Program
}

${ }^{2}$ Department of Global Health, Academic Medical Centre, University of Amsterdam, Amsterdam Institute for Global

\begin{abstract}
Engagement of the private sector and community leaders are popular strategies for improving tuberculosis (TB) case detection. However the impact of engaging pharmacies and community shop keepers in TB referral varies and the best method to achieve it are unknown.

To evaluate changes in referral and TB case detection following workshops and/or motivational "detailing" visits, mystery clients visited 188 pharmacies and 103 grocery stores to seek advice for chronic symptoms reflecting pulmonary TB. Most sites $(89 \% \mathrm{n}=255)$ were exposed to "detailing" and $26.8 \%(76)$ also attended 1 hour workshops designed by the National TB program and KNCV Tuberculosis Foundation (KNCV). Sites were scored pre- and post-intervention, obtaining 1 point each for a) spontaneous recognition of TB symptoms, b) acknowledgement of symptoms as possibly TB when prompted, c) referral to medical care d) not promoting drugs e) exhibiting TB health education materials on site and f) referral of mystery clients to the TB program.

The TB recognition and referral scores of all exposed establishments increased by an average of 2.0 points (95\%C.I. 1.92.1 ) at post-test compared with an increase of 0.6 points (95\%C.I. 0.4-.0.9) among those in the comparison group $(\mathrm{p}<0.001)$. Attempted sales of antibiotics and palliatives declined from $38.3 \%$ at baseline to $16.1 \%$. After six months, $33.1 \%$ of pharmacies and $22.7 \%$ of grocery stores reported referring. Seven smear-positive TB cases were diagnosed from among 70 TB suspects referred by pharmacies, but none from among 30 suspects sent by groceries. TB investigations rose by $8.4 \%$ in the area for a six month period. The intervention is likely to have contributed to the doubling of TB case notifications over two years.
\end{abstract}

A simple motivation intervention led to improvement in referral and TB case detection. The National TB program introduced interventions with pharmacies to other provinces.

Keywords: Pharmacies, tuberculosis, public-private partnerships, referral, Dominican Republic, KNCV.

\section{INTRODUCTION}

Engagement of the private sector is a recommended strategy for increasing TB case detection and managing treatment. Efforts began tentatively through partnerships with private physicians, but have gained momentum globally as more TB programs engage pharmacists, treatment activists, traditional healers, labor unions, faith-based organizations, small businesses, and representatives of atrisk and vulnerable groups.

*Address correspondence to this author at the $\mathrm{KNCV}$ Tuberculosis Foundation, PO Box 146, 2501 CC The Hague, The Netherlands; Tel: +31 7041672 74; Fax: +31 7035840 04; E-mail: mitchelle@kncvtbc.nl
In the Dominican Republic, the need for stronger community linkages and new partnerships stems from the dynamics of the TB epidemic. The poor are more likely to use informal services to meet their health care needs and to allocate a higher proportion of health care spending on medication, making pharmacies and corner stores logical partners in early TB detection [1].

TB incidence is less than $100 / 100,000$ population and is increasingly concentrated in persons co-infected with Human Immunodeficiency Virus (HIV) and in marginalized and vulnerable groups, including Haitian labor migrants, sex workers, alcohol and drug users, and prisoners [2, 3]. Understanding of TB is most limited in the country's poorest 
quintile, with only $57 \%$ of women and $51 \%$ of men aware that $\mathrm{TB}$ can be cured, and only $47 \%$ and $35 \%$ cognizant of its airborne transmission [1].

Globally pharmacies and small neighborhood shops that sell medicines informally are often envisioned as obstacles to TB control because they often dispense palliative treatments, mono-therapies, or insufficient dosages that contribute to the rise of multi-drug resistance [4-9]. However, efforts to promote referral by pharmacies and small businesses in Bolivia, India, Nepal, Vietnam, Malawi and elsewhere have shown modest successes in enhancing timely referral to $\mathrm{TB}$ care and reducing the unregulated sale of TB medications [10-14]. Moreover pharmacists often have unique access to certain vulnerable groups, such as diabetics $[15,16]$. Consequently in many contexts pharmacies are playing an important role in screening, tuberculin skin test screening and direct observation of TB treatment [12, 17-23].

Community grocery stores have been less engaged in TB control efforts but have been effective in osteoporosis screening and other public health referral efforts [24-26]. In Santo Domingo, small grocery stores, called colmados, are important social spaces where neighbors meet to exchange news, alcohol is often consumed, diverse licit and illicit transactions occur. Informal community "bars" or drinking establishments have long been known to serve as TB transmission hot spots in many settings [27-31].

This study measured the effectiveness of two strategies to motivate referral of TB suspects by private pharmacies and neighborhood corner stores (colmados). Specifically, we sought to measure changes in a) vendors' recognition of TB symptoms $b$ ) number of referrals to the TB program and c) the number of TB suspects investigated by the TB program in the catchment area following educational workshops and/or detailing visits.

\section{TECHNICAL ASSISTANCE}

The TB program of the Dominican Republic requested KNCV Tuberculosis Foundation (KNCV) to develop a project supported by the United States Agency for International Development (USAID) with innovative interventions responding to the need to increase early case finding among the most difficult to reach populations. One of the project strategies developed by $\mathrm{KNCV}$ was a participatory and volunteer-based approach to engage groups outside the formal health sector in the fight against TB. A team of stakeholders from $\mathrm{KNCV}$ headquarters and local office, TB program staff, and a local research institute National Center for The Study of Maternal-Child Health (CENISMI) worked together, drawing from experiences in other countries and the growing literature. CENISMI had a successful history of collaboration on TB/HIV monitoring and evaluation and therefore took the lead in conducting the impact evaluation with support from the Research Unit of KNCV's headquarters in The Hague.

\section{METHODS}

A quasi-experimental design was intended, but implementers allocated more sites to the intervention arm than specified, in order to leverage the potential benefit of improved TB detection. Thus, a pragmatic trial design with unequal-sized study arms and non-random allocation was implemented.

The protocol was approved by the ethical review committee of the CENISMI and a waiver to conduct simulations without prior informed consent was granted due to the negligible risk of social or physical harms.

\section{Setting}

The study took place in Area IV, a neighborhood 18.42 $\mathrm{km}^{2}$ in size with a population of 489,296 inhabitants located in the center in the capital city. The notified TB rate of Area IV was $99 / 100,000$ population in 2009 making it a hot spot. (see Fig. 1).

\section{The Sample}

Pharmacy inclusion criteria included both registration as a pharmacy and location in the catchment area (Area IV). Exclusion criteria were those pharmacies that sold only to wholesale distributors and those located within public hospitals. A census of operating pharmacies was undertaken

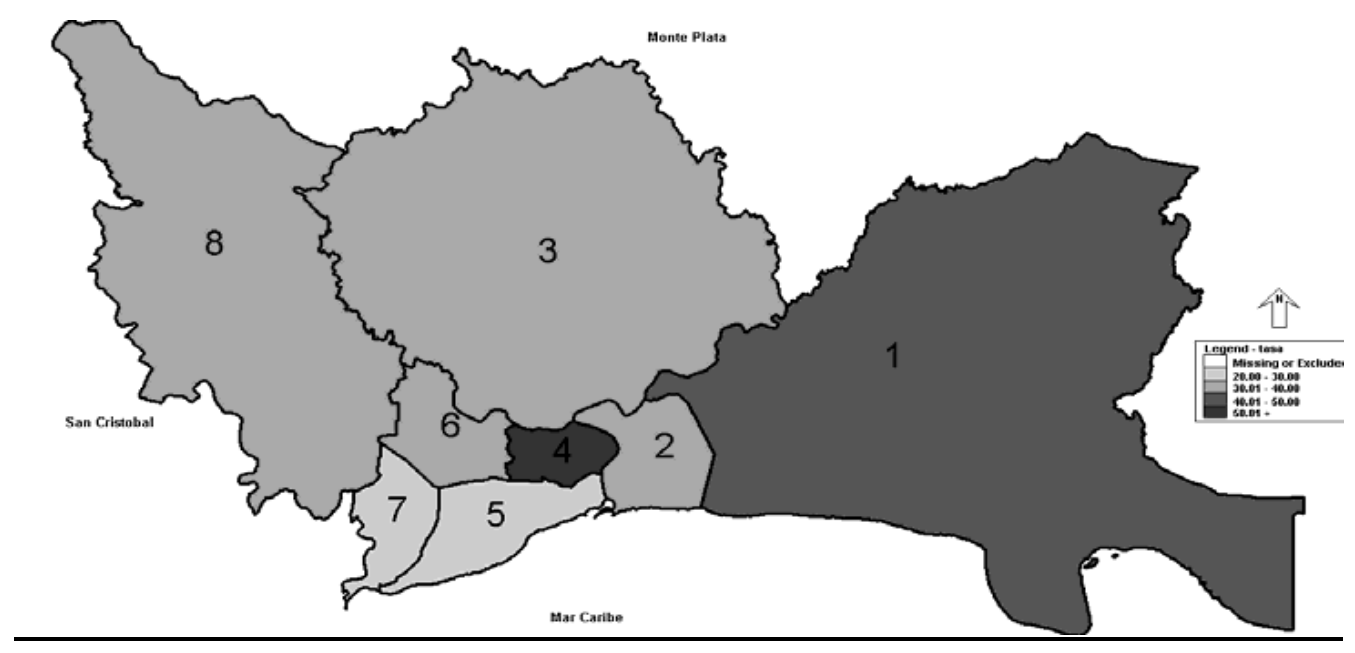

Source: Ministry of Health (SESPAS), used with permission

Fig. (1). Notified TB Rates (per 100,000) in the Health Areas of Santo Domingo, Dominican Republic. 

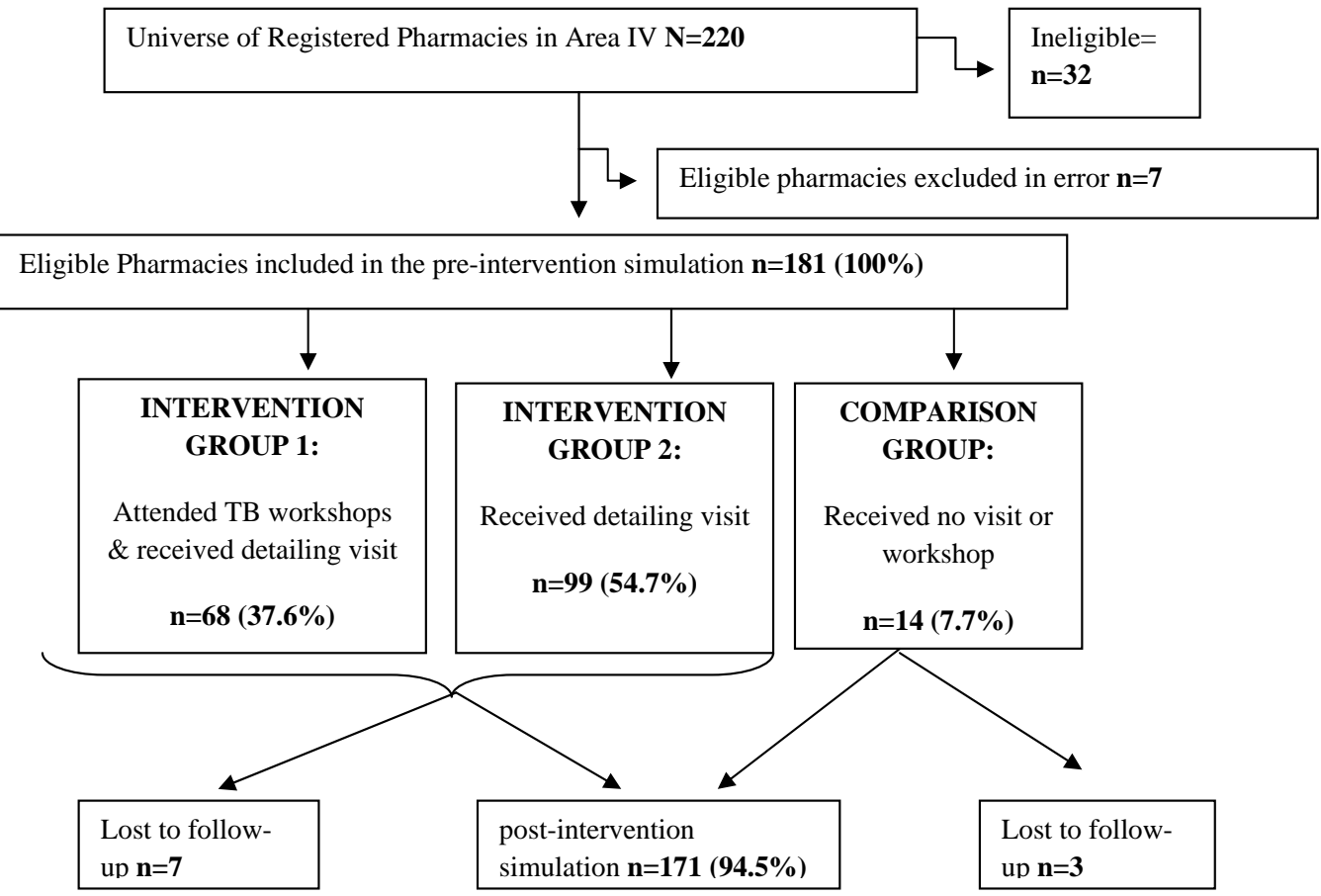

Fig. (2). Disposition of the pharmacy sample.

by the municipal health services to develop the sampling frame. Of the 220 pharmacies identified in November, 2009, a total of $188(85.5 \%)$ met all study inclusion criteria and 181 (96.3\%) were included (Fig. 2).

Developing a sampling frame of corner grocery stores was challenging since they do not require sanitary registration and liquor distributors would not share their proprietary customer lists. So purposive quota sampling was employed by purposefully selecting 5-8 corner stores in each of the 13 neighborhoods in the catchment area (see Fig. 3). Stores in under-served areas were preferentially selected, meaning that they were not within 500 meters of pharmacies or hospitals.

\section{The Interventions}

$\mathrm{KNCV}$ and the national TB program designed a strategy to educate vendors about the symptoms of $\mathrm{TB}$ and to motivate referral of customers with chronic TB symptoms. The two interventions consisted of either: a) an educational workshop plus a motivational 'detailing visit' or b) a 'detailing visit(s)' alone. Historically, academic detailing visits were brief marketing conversations initiated by ambulatory commercial pharmaceutical salespersons (aka "drug reps") who sought to engage clinicians or pharmacists in interactive educational dialogs with the goal of promoting the prescription or sale of specific products and often involve distribution of drug samples and/or branded materials [32]. These commercial visits have been shown to influence prescribing and dispensing behaviors [32-35]. Given their distorting effect, these visits have been banned in some settings. However, academic detailing has been reengineered and adapted for public health and social marketing purposes [36]. Educational outreach visits to vendors have been shown to reduce misuse of antibiotics $[37,38]$.
Trained health educators from the Santo Domingo office of KNCV and the National TB program implemented the intervention through both modalities jointly. Both modalities presented the same content: importance of early recognition of TB symptoms, diagnosis of TB, TB treatment free of charge, and the high probability of cure with treatment adherence. Facilities received low literacy posters, brochures, referral registers, calendars with TB messages, and referral slips to stimulate referrals.

Workshops were held in community settings (e.g. churches, health centers) and consisted of a one hour, low literacy, audiovisual presentation on TB symptoms and the important role to be played by community businesses. It was followed by a participatory dialog by KNCV staff and volunteers intended to reinforce social norms of solidarity and the importance of social entrepreneurship to improve the community's health. Workshop participants were invited to demonstrate their commitment to referral, awareness-raising, and the fight against TB by signing a pledge ("Mi Compromiso"). Participants received a certificate of recognition to display, to heighten positive peer reinforcement of their contribution. Supportive IEC materials (a pharmacy and colmado poster and TB leaflet dispensers) were designed for the pharmacies and colmados involved in the intervention.

Due to the larger relative size of pharmacies, each received two detailing visits, whereas grocery stores received only one visit. The initial visits lasted an average of 16 minutes (Interquartile range (IQR) 10-20), with an average of 11 minutes dedicated to TB content. Follow-up booster visits at pharmacies lasted an average of 7 minutes (IQR 411). A team of 8 TB program staff and volunteers conducted and average of 18 detailing visits per day (IQR 22-26). 


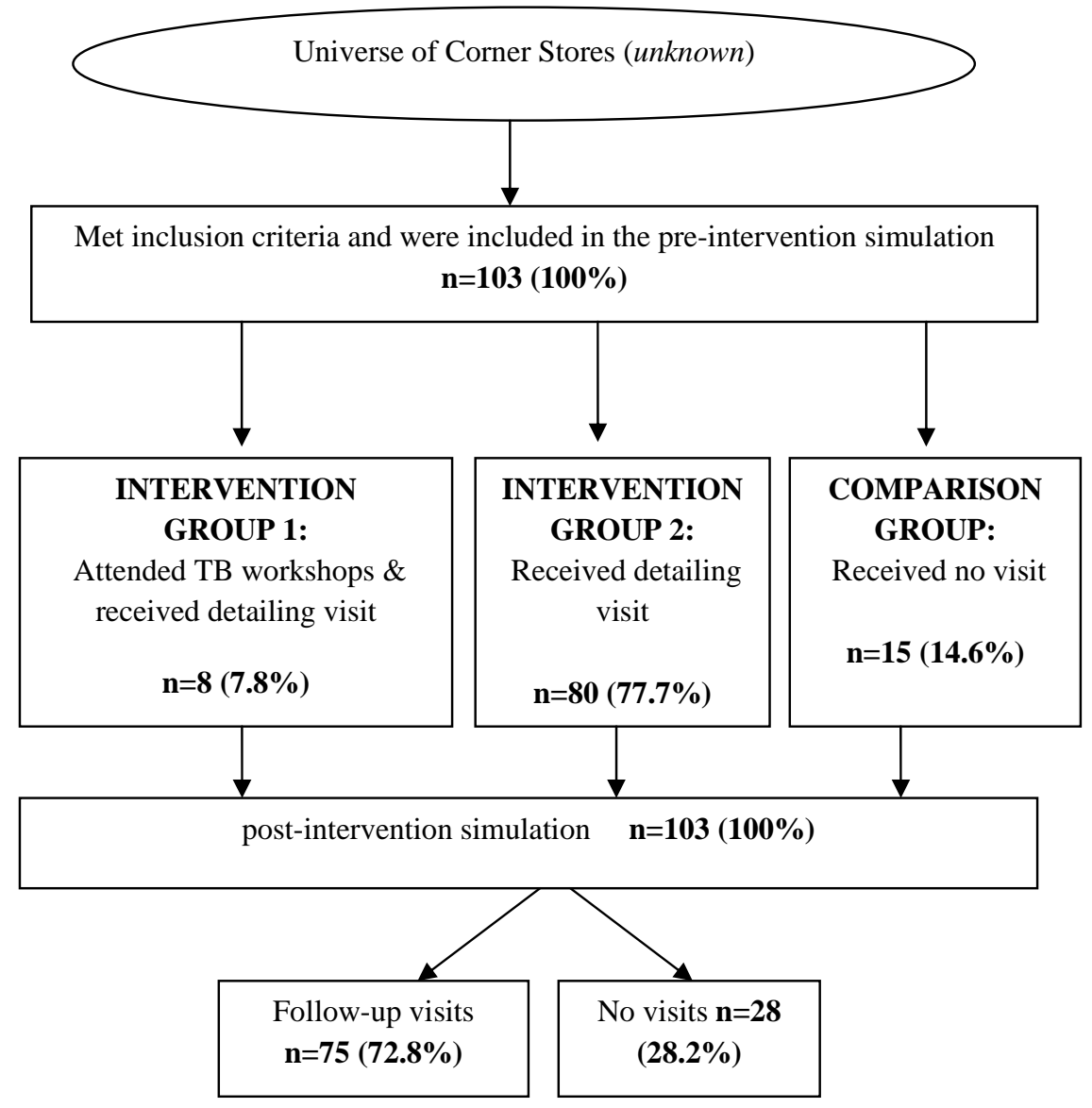

Fig. (3). Disposition of the corner store sample.

All sites in the intervention group were invited to TB workshops, however acceptance of the workshop modality among vendors was low, with only $68(37.6 \%)$ pharmacies and eight corner stores (7.8\%) participating (See Figs. 1, 2). After 4 weeks, the face to face workshop modality was replaced by the detailing visits to increase feasibility and acceptability of the educational intervention. Two "comparison" groups of 14 pharmacies and 15 corner stores were created circumstantially when detailing visits could not be completed due to limited access during flooding, temporary closure, gang activity, or other logistical constraints.

\section{Measurement}

Pre-intervention "mystery clients" trained by CENISMI, an independent research team, were sent to the sites an average of three to six weeks before the interventions began and again two to six weeks afterwards. Trained actors approached vendors reporting a set of chronic TB symptoms, having received no relief from the use of cold medicines, and asked for advice. After the staff had given an initial response, the actor suggested that perhaps the symptoms might be tuberculosis-related and asked the staff person for their view on this idea. After the actor left the establishment, he/she completed a data capture form noting whether TB symptoms were recognized (either spontaneously or after prompting), whether any referral was given, whether a referral was TB-program specific, whether any sale of medication was attempted, and if so what product was promoted. Then the actors recorded subjective impressions of the encounter, the verbatim responses, non-verbal reactions, and mitigating circumstances. For convenience, the same actor often conducted both simulations four months apart.

Finally, follow-up visits were conducted by the TB to the pharmacies to drop off referral books and educational materials and reinforce the main messages. After six months, a final visit was made to review the on-site TB referral logs kept by businesses and to measure the volume of referral of actual TB suspects. Referral logs were compared with the referral slips collected at health facilities to determine the volume and proportion of effective referrals. Names on TB referral slips and logs were compared against the TB patient register to identify TB cases detected as a consequence of referrals from either pharmacies or stores.

\section{Analysis}

An unweighted score of $6 \mathrm{~TB}$ behaviors was constructed. Pharmacies received 1 point each for a) spontaneous recognition of TB symptoms b) recognition of TB when prompted c) recommendation to seek medical care d) not attempting to sell products to chronic coughers e) having TB health education materials on display and $\mathrm{f}$ ) referring to a specific facility where TB care is rendered. Open-ended questions on referral and types of recommended medications were coded by a single analyst using pre-specified criteria. Alpha was set at 0.05 . 


\section{RESULTS}

At baseline, establishments were unlikely to encourage customers reporting chronic cough, fever and weight loss to consider TB, suggesting instead common ailments such as cold, flu, diabetes, malaria, heat exhaustion, and folk illnesses due to abrupt weather-changes. As Table 1 shows, the TB recognition and referral scores of all exposed establishments increased by an average of 2.0 points ( $95 \% \mathrm{CI}$ : $1.9-2.1)$ at posttest compared with an increase of 0.6 points $(95 \% \mathrm{CI}$ : 0.4-$.0 .9)$ among those in the comparison group $(\mathrm{p}<0.001)$. Pharmacies exposed to the intervention improved by 2.12 on average compared with an improvement of 0.9 in the comparison group $(\mathrm{p}=0.06)$ and groceries stores in the intervention arm yielded an average improvement of 1.7 points (95\%CI: $1.5-1.9)$ versus 0.4 (95\%CI: 0.1-0.7) among those without exposure $(\mathrm{p}=0.006)$. There was no demonstrable dosage effect between those receiving only detailing visits whose scores increased 1.9 points (95\%CI: $1.8-2.0)$ and those with the additional workshop exposure who improved an average of 2.18 points $(95 \% \mathrm{CI}: 2.0-2.4)(\mathrm{p}=.06)$.

Almost half $(45.0 \%)$ of pharmacies in the intervention group were displaying TB health information on the premises at post-test as compared with only $9 \%$ of comparison sites. A similar proportion $(49.7 \%)$ of intervention pharmacies referred mystery clients directly to the national TB program when chronic TB symptoms were described whereas comparison sites referred only $18.2 \%$ of mystery clients. All the intended behaviors improved at post-test, however some gains were modest. At baseline, 64 (38.3\%) pharmacy simulations prompted an attempt to sell a medicine including antibiotics without a prescription (e.g. amoxicillin, cephalosporin, rifampicin, and cefaplus ${ }^{\mathrm{TM}}$ ). After the intervention, cold remedy sales attempts declined to $16.1 \%$. In the second simulation there were no attempts to dispense antibiotics without a prescription in response to chronic TB symptoms.

Corner stores exposed to detailing and workshops also improved their performance in every $\mathrm{TB}$ recognition and referral category at post-test. However only acceptance of TB as a potential diagnosis when prompted $(6.8 \%$ to $48.3 \%)$, referrals to medical care generally $(30.7 \%$ to $59.8 \%)$, and referral to the TB program specifically (3.4\% to $36.4 \%$ ) improved significantly.

Corner stores are not authorized to sell pharmaceuticals and as expected, visited corner stores sought to sell few medicines at baseline (18.2\%). At baseline, corner stores frequently advocated self-medication, referring those reporting chronic TB symptoms to pharmacies instead of health care facilities. Medicine sales attempts declined at post-test $(8.5 \%)$, however, ciprofloxin was offered by one grocery.
At follow-up six months later, among 220 sites (79\%) visited by TB educators, $33.1 \%$ of pharmacies (48/145) and $22.7 \%$ of corner stores $(17 / 75)$ reported having referred 70 and 30 individual TB suspects respectively. Of these 100 referred suspects investigated over 6 months, 7 cases (7\%) of smear positive pulmonary TB were diagnosed, all from pharmacy referrals. In the two year period following the intervention, detection of new smear positive cases averaged 150 per quarter, as compared with 67 per quarter in the preintervention period (See Fig. 4). Interestingly, TB investigations rose by $8.4 \%$ in the two quarters following the intervention, but increases were not sustained over time and may have even declined in 2012 (data are incomplete).

\section{DISCUSSION}

The intervention appears to improve TB symptom recognition and referral behaviors among vendors in private sector pharmacies and groceries. This was consistently shown in both simulations as well as through actual referral for TB screening by participating businesses in the catchment area. The intervention appeared to reduce the attempted sale of antibiotics without a prescription in response to chronic TB symptoms. Post-test simulations were completed within a month of the intervention and thus the duration of intervention effect is unknown. Aggregate increases in TB detection in the catchment area were noted for up to two years post intervention but increases in $\mathrm{TB}$ investigations lasted only six months (Fig. 4).

Pragmatic trial designs such as this one can enhance cooperation and ownership by TB control leadership responsible for improving detection and treatment of TB patients in the short term. Indeed the national TB program chose to scale up the intervention in several provinces following this trial. However non-random and disproportionate allocation to treatment arms entails sacrifices of both statistical power and precision that international technical assistance organizations may find hard to defend. Striking the right balance between the pragmatic public health and safety goals of TB control managers and the design standards of TB researchers requires compromises. A pre and post design helped to mitigate some of the concerns about bias that non-random allocation caused.

\section{LIMITATIONS}

This evaluation has limitations. Most importantly, KNCV conducted social mobilization efforts in the catchment area which likely contributed to the large increases in overall TB detection. Allocation to treatment groups was non-random and potential unidentified differences in the establishments cannot be ruled out. The fact that sites received varying dosages of the intervention (workshops and/or detailing) makes the attribution of effect more challenging. The unequal sizes of the treatment arms resulted in limited power

Table 1. The Differences in Difference within and Between Study Arms

\begin{tabular}{|c|c|c|c|c|c|c|c|c|}
\hline \multirow{2}{*}{ Item } & \multicolumn{3}{|c|}{ Pharmacies } & \multicolumn{3}{c|}{ Corner Stores } & \multicolumn{2}{c|}{ Overall Effect* } \\
\cline { 2 - 9 } & Pre & Post & Difference (95\% CI) & Pre & Post & Difference (95\% CI) & Difference (95\% CI) & p-Value \\
\hline \hline Intervention & 1.5 & 3.6 & $2.1(2.0-2.2)$ & 1.2 & 2.9 & $1.7(1.5-1.9)$ & $2.0(1.9-2.1)$ & $<0.001$ \\
\hline Comparison & 1.6 & 2.5 & $0.9(0.4-1.3)$ & 1.1 & 1.5 & $0.4(0.1-0.7)$ & $0.6(0.4-.0 .9)$ & \\
\hline
\end{tabular}

*Both intervention groups combined and both control groups combined. 


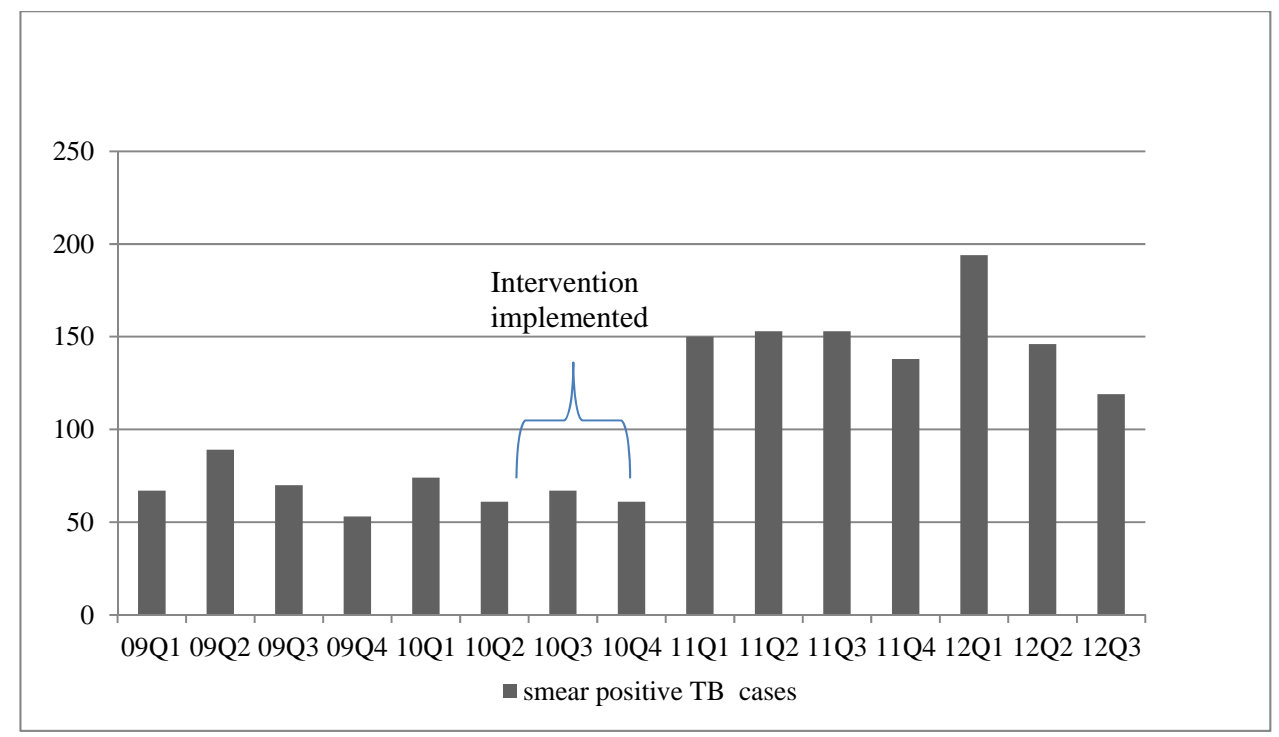

Fig. (4). Detection of Smear Positive TB in Area IV by Quarter 2009-2012.

to detect small differences and a probable underestimate of the true effectiveness of the interventions. This was compounded by three pharmacy closures in the "comparison arm" further reducing study power (from 14 to 11 sites). Thus the study lacked the power to assess the marginal effect of more intense exposure. Review of the qualitative observational data indicated that many pharmacy and corner store staff did not always believe that the actors had tuberculosis symptoms because the actors appeared robust and healthy. Future studies may prefer to employ underweight actors with demonstrable malaise to better simulate active TB disease. This design flaw may have lead to an underestimation of the efficacy of the intervention.

TB control efforts in moderate incidence settings like the Dominican Republic require a tailored approach toward communities who have historically underutilized TB services and experienced barriers to high quality care. Small businesses appear both willing and able to contribute to the early detection of tuberculosis in poor urban communities if given the tools to do so. Referrals from pharmacies and corner stores prompted by a short and simple motivational visit helped the national TB program to find seven new smear-positive TB cases in the first six months and likely contributed to the doubling in TB case detection over the previous year.

Further research is needed to see if this approach reaches an under-served self-medicating segment of the population that would not have been reached through passive detection, or if detailing simply accelerates the timely detection of cases that would eventually be identified through the normal program routines. This issue of the "additionality" is important to international TB technical assistance organizations and TB programs alike, as both seek to maximize gain from dwindling resources.

\section{CONCLUSION}

To reduce delay in care seeking for chronic TB symptoms, KNCV Tuberculosis Foundation and the National TB program partnered to develop an innovative community-based publicprivate strategy to reach under-served and vulnerable groups. Involvement of pharmacy and corner stores appears to increase
TB case detection, and reduce promotion of antibiotics without a prescription. Approaches such as this should be rigorously tested and only the demonstrably effective approaches brought to scale. Future operational research should explore the duration of effect of detailing, the impact of booster visits, additionality, varying dosages of intervention, and the cost effectiveness of visits vis-á-vis passive case finding.

\section{ABBREVIATIONS \\ CENISMI = Centro Nacional de Investigaciones en Salud Materna Infantil \\ HIV = Human immunodeficiency virus \\ SESPAS $=$ Ministry of Public Health Dominican Republic \\ TB $=$ tuberculosis \\ USAID = United States Agency for International Development}

\section{CONFLICT OF INTEREST}

The authors confirm that this article content has no conflict of interest.

\section{ACKNOWLEDGEMENTS}

We are indebted to the actors, the pharmacy staff, the corner grocery store owners, and TB education volunteers for their collaboration. This operations research project was funded the TBCAP project of USAID but the views expressed are only attributable to the authors.

\section{REFERENCES}

[1] CESDEM. Encuesta Demográfica y de Salud 2007. Santo Domingo, República Dominicana: Centro de Estudios Sociales y Demográficos (CESDEM) y Macro International Inc. 2008.

[2] Baez JP-TE, Acosta I, Elias-Melgen R, et al. HIV and tuberculosis coinfection in the Dominican Republic. Proceedings of the 37th Union World Conference on Lung Health; 2006 Oct-Nov; Paris, France. Abstract No. 61528, 2006.

[3] Perez-Then E BJ, Acosta I, Elias-Melgen R, Marcelino B, Tavarez M et al. Clinical profile for tuberculosis patients co-infected with HIV in the Dominican Republic. Proceedings of the 37th Union World Conference 
on Lung Health; 2006 Oct-Nov; Paris, France. Abstract No. 61521, 2006.

[4] Lambert ML, Delgado R, Michaux G, et al. Tuberculosis control and the private health sector in Bolivia: a survey of pharmacies. Int J Tuberc Lung Dis 2004; 8: 1325-9.

[5] Rojpibulstit M, Kanjanakiritamrong J, Chongsuvivatwong V. Patient and health system delays in the diagnosis of tuberculosis in Southern Thailand after health care reform. Int J Tuberc Lung Dis 2006; 10: 4228.

[6] Kiwuwa MS, Charles K, Harriet MK. Patient and health service delay in pulmonary tuberculosis patients attending a referral hospital: a crosssectional study. BMC Public Health 2005; 5: 122

[7] van den Boogaard J, Semvua $\mathrm{HH}$, Boeree MJ, et al. Sale of fluoroquinolones in northern Tanzania: a potential threat for fluoroquinolone use in tuberculosis treatment. J Antimicrob Chemother 2010; 65: 145-7.

[8] Hurtig AK, Pande SB, Baral SC, et al. Linking private and public sectors in tuberculosis treatment in Kathmandu Valley, Nepal. Health Policy Plan 2002; 17: 78-89.

[9] Rojpibulstit M, Kanjanakiritamrong J, Chongsuvivatwong V. Patient and health system delays in the diagnosis of tuberculosis in Southern Thailand after health care reform. Int J Tuberc Lung Dis 2006; 10: 4228.

[10] Rajeswari R, Balasubramanian R, Bose MSC, et al. Private pharmacies in tuberculosis control - a neglected link. Int J Tuberc Lung Dis 2002; 6 : 171-3.

[11] Lonnroth K, Karlsson M, Lan NTN, et al. Referring TB suspects from private pharmacies to the National Tuberculosis Programme: experiences from two districts in Ho Chi Minh City, Vietnam. Int J Tuberc Lung Dis 2003; 7: 1147-53.

[12] Lambert ML, Delgado R, Michaux G, et al. Collaboration between private pharmacies and national tuberculosis programme: an intervention in Bolivia. Trop Med Int Health 2005; 10: 246-50.

[13] Newell JN, Pande SB, Baral SC, et al. Control of tuberculosis in an urban setting in Nepal: public-private partnership. Bull World Health Org 2004; 82: 92-8.

[14] Vu DH, van Rein N, Cobelens FG, et al. Suspected tuberculosis case detection and referral in private pharmacies in Viet Nam. Int $\mathbf{J}$ Tuberc Lung Dis 2012; 16: 1625-9.

[15] Gnanasan S, Ting KN, Wong KT, et al. Convergence of tuberculosis and diabetes mellitus: time to individualise pharmaceutical care. Int $\mathrm{J}$ Clin Pharm 2011; 33: 44-52.

[16] Lonnroth K, Thuong LM, Linh PD, et al. Utilization of private and public health-care providers for tuberculosis symptoms in Ho Chi Minh City, Vietnam. Health Policy Plan 2001; 16: 47-54.

[17] Tavitian SM, Spalek VH, Bailey RP. A pharmacist-managed clinic for treatment of latent tuberculosis infection in health care workers. Am J Health Syst Pharm 2003; 60: 1856-61.

[18] Juan G, Lloret T, Perez C, et al. Directly observed treatment for tuberculosis in pharmacies compared with self-administered therapy in Spain. Int J Tuberc Lung Dis 2006; 10: 215-21.

[19] Hess K, Goad J, Wu JN, et al. Isoniazid Completion Rates for Latent Tuberculosis Infection Among College Students Managed by a Community Pharmacist. J Am College Health 2009; 57: 553-5.
[20] Smith F. Private local pharmacies in low- and middle-income countries: a review of interventions to enhance their role in public health. Trop Med Int Health 2009; 14: 362-72.

[21] Hecox N. Tuberculin skin testing by pharmacists in a grocery store setting. J Am Pharm Assoc 2003; 48: 86-91.

[22] Clark PM, Karagoz T, Apikoglu-Rabus S, et al. Effect of pharmacistled patient education on adherence to tuberculosis treatment. Am J Health Syst Pharm 2007; 64: 497-506.

[23] Lonnroth K, Lambregts K, Nhien DTT, et al. Private pharmacies and tuberculosis control: A survey of case detection skills and reported antituberculosis drug dispensing in private pharmacies in Ho Chi Minh City, Vietnam. Int J Tuberc Lung Dis 2000; 4: 1052-9.

[24] Lai LK, Armstrong EP, Salcido AL, et al. Women's perception of bonemineral density testing and osteoporosis education in the community pharmacy. Consult Pharm 2003; 18: 968-76.

[25] Goode JV, Swiger K, Bluml BM. Regional osteoporosis screening, referral, and monitoring program in community pharmacies: findings from Project ImPACT: Osteoporosis. J Am Pharm Assoc 2003; 44 $152-60$

[26] Hecox N. Tuberculin skin testing by pharmacists in a grocery store setting. J Am Pharm Assoc 2008; 48: 86-91.

[27] Morojele NK, Kachieng'a MA, Mokoko E, et al. Alcohol use and sexual behaviour among risky drinkers and bar and shebeen patrons in Gauteng province, South Africa. Soc Sci Med 2006; 62: 217-27.

[28] Macintyre K, Bloss E. Alcohol brewing and the african tuberculosis epidemic. Med Anthropol 2011;30: 126-35.

[29] Moller V, Erstad I, Zani D. Drinking, smoking, and morality: do 'drinkers and smokers' constitute a stigmatised stereotype or a real TB risk factor in the time of HIV/AIDS? Soc Indic Res 2010; 98: 217-38.

[30] Kalichman SC, Simbayi LC, Vermaak R, et al. HIV/AIDS risks among men and women who drink at informal alcohol serving establishments (shebeens) in Cape Town, South Africa. Prev Sci 2008; 9: 55-62.

[31] Murray EJ, Marais BJ, Mans G, et al. A multidisciplinary method to map potential tuberculosis transmission 'hot spots' in high-burden communities. Int J Tuberc Lung Dis 2009; 13: 767-74.

[32] Bhargava V, Greg ME, Shields MC. Addition of Generic Medication Vouchers to a Pharmacist Academic Detailing Program: Effects on the Generic Dispensing Ratio in a Physician-Hospital Organization. J Manag Care Pharm 2010; 16: 384-92.

[33] Gonul FF, Carter FJ. Impact of e-detailing on the number of new prescriptions. Health Care Manag Sci 2010; 13: 101-11.

[34] Loganathan M, Singh S, Franklin BD, et al. Interventions to optimise prescribing in care homes: systematic review. Age Ageing 2011; 40: $150-62$.

[35] Chauhan D, Mason A. Factors affecting the uptake of new medicines in secondary care - a literature review. J Clin Pharm Ther 2008; 33: 33948.

[36] Fischer MA, Avorn J. Academic detailing can play a key role in assessing and implementing comparative effectiveness research findings. Health Aff 2012;31: 2206-12.

[37] Wesson J, Olawo A, Bukusi V, et al. Reaching providers is not enough to increase IUD use: A factorial experiment of 'academic detailing' in Kenya. J Biosoc Sci 2008; 40: 69-82.

[38] Arnold SR, Straus SE. Interventions to improve antibiotic prescribing practices in ambulatory care. Cochrane Database Syst Rev 2005;19: CD003539. 